\title{
Strategic Participation of Merchant Energy Storage in Joint Energy-Reserve and Balancing Markets
}

\author{
Arthur Schillemans \\ BCG \\ Brussels, Belgium \\ schillemans.arthur@bcg.com
}

\author{
Gustavo De Vivero Serrano \\ KU Leuven \& EnergyVille \\ Leuven \& Genk, Belgium \\ gustavoadan.deviveroserrano@kuleuven.be
}

\author{
Kenneth Bruninx \\ KU Leuven, VITO \& EnergyVille \\ Leuven, Mol \& Genk \\ kenneth.bruninx@kuleuven.be
}

\begin{abstract}
Energy storage systems (ESS) may provide the required flexibility to cost-effectively integrate stochastic electricity generation from renewable energy sources. Energy storage owners should, however, be properly renumerated for the services they provide. In this paper, we propose a bilevel optimization problem, mimicking the strategic behavior of a price-making ESS owner in a joint day-ahead (DA) energy-reserve market. We explicitly consider possible real-time balancing market outcomes when clearing the DA markets via probabilistic reserve constraints and ensure the feasibility of dispatching ESS-based reserves through novel worst-case reserve activation constraints. The proposed framework may be used by regulators and market operators to assess market design options or by ESS owners to optimize their bids.
\end{abstract}

Index Terms-Energy Storage, Joint Energy-Reserve Market, Balancing Market, Bilevel Optimization, Mathematical Problem with Equilibrium Constraints

\section{INTRODUCTION}

Energy Storage Systems (ESS) may play a pivotal role in the transition to power systems with high shares of intermittent electricity generation from renewable energy sources (RES) [1]. This drives a growing interest on, i.a., ESS' participation in electricity markets [2], business models [3], ESS siting \& sizing [4], ownership structures [5] and distributed ESS [6].

Energy storage owners may base their business models on, i.a., (1) price arbitrage in the energy market and (2) providing ancillary services such as operating reserves. However, the possible bids of an ESS owner are not only constrained by its installed capacity (MW), as for conventional generation capacity, but also by its current and anticipated storage content and capacity (MWh) [7]. Not accounting for these inter-temporal and inter-service (energy-operating reserves) constraints may lead to overly optimistic bid strategies, e.g., offering regulating services that the ESS is unable to deliver. These constraints, however, significantly increase the complexity of the bid problem of a strategic ESS owner participating in a joint energy-reserve market.

Contreras-Ocana et al. [6] study a price-taking ESS owner participating in joint energy-reserve markets. Similarly, Zou et al. [8] consider the ESS as a price-maker in the energy market and as price-taker in the operating reserve market. However, these authors disregard the potential risk of non-delivery due to the activation of these reserves in real-time. To overcome this issue, several authors, such as [9]-[12] (price-taking ESS) and
[13], [14] (price-making ESS), introduced balancing markets in the context of strategic ESS participation in joint energyreserve markets. However, the real-time availability of the ESS-based operating reserves is not guaranteed. Nasrolahpour et al. [14] account for the expected activation of reserves averaged over a set of scenarios, which does not guarantee feasibility across all possible reserve activation scenarios, or explicitly enforce state of charge constraints in each reserve activation scenario at a significant computational cost. In addition, [13], [14] propose a separate model for the joint energy-reserve market and the balancing market, which does not account for the trade-offs between these markets.

In this paper, we propose a bilevel model for a price-making ESS owner participating in a joint DA energy-operating reserves market and a real-time (RT) balancing market. The contributions of our work are twofold. First, the market clearing problem, governing the ESS owner's expectation on the energy, reserve and balancing market outcome, represents the joint minimization of operating costs in these markets. The uncertainty on real-time imbalances is represented via a set of probabilistic reserve constraints, inspired by [15], which allow considering possible balancing market outcomes and costs while clearing the joint energy-reserve market. This enables the ESS owner to correctly assess its expected revenue across markets. Second, the real-time availability of ESS-based operating reserves is guaranteed by including a set of worst-case reserve activation constraints, inspired by [7]. Although this worst-case approach typically yields more conservative bids than scenario-based approaches [13], [14], the additional computational cost is negligible compared to the computational cost associated with scenario-based approaches [7]. Furthermore, scenario-based approaches only guarantee feasibility of activating the ESS-based reserves in the considered scenarios, whereas our approach covers all possible activation scenarios. The combination of probabilistic reserve constraints to account for real-time imbalances and these worst-case constraints effectively decouples the feasibility and optimality, as opposed to scenario-based approaches.

The remainder of this paper is structured as follows. In Section II, we introduce the bilevel optimization problem. A case study, illustrating the benefits of the proposed model, is presented in Section III. Section IV contains concluding remarks and suggestions for future work. 


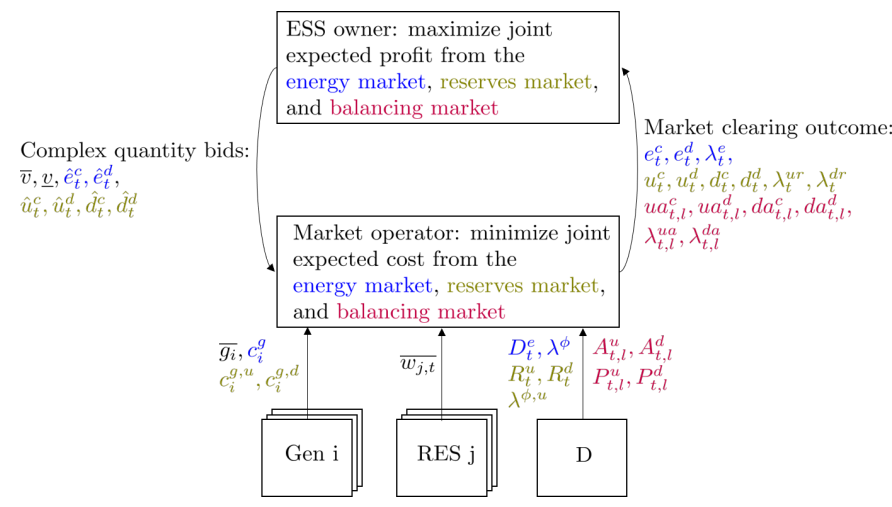

Fig. 1. The energy storage system (ESS) owner maximizes the expected profit from the energy, reserve and balancing market by taking strategic positions via complex quantity bids in the energy and reserve market (upper level problem). These bids are determined based on the ESS owner's expectation of the market clearing outcomes, as represented in the lower level market operator's problem, in which the sum of the electricity generation cost in the DA market, the reserve procurement cost and the expected reserve activation cost is minimized.

\section{Methodology}

First, we introduce the profit-maximization problem the strategic energy storage owner faces and how it forms its expectation of the energy, reserve and balancing market outcomes (Section II-A). In Section II-B, the notation used in this paper is introduced. Third, we provide the mathematical formulation of the proposed bilevel optimization problem and the reformulation of the resulting mathematical problem with equilibrium constraints (MPEC) as a mixed integer linear programming (MILP) problem (Section II-C).

\section{A. Description of the bilevel problem}

A single price-making agent, owning an ESS, participates in the DA and balancing markets to maximize its profit. The associated bilevel model, resembling a Stackelberg Game [16], consists of an upper-level (UL) problem where the the ESS decides on its offering strategy to maximize. The ESS owner anticipates the impact of these decisions by considering a lower level (LL) joint energy-reserve and balancing market clearing problem (Fig. 1). This bilevel approach allows endogenously determining both the ESS owner's offering strategy and the resulting clearing prices.

1) Upper-level problem: the energy storage owner's bid: In the UL problem, the ESS owner maximizes his expected joint profit from participation in the energy, reserve, and balancing markets by formulating complex quantity bids [2]. These bids contain feasible energy (dis)charge and reserve capacity quantities for each time step, and the ESS's technical limitations which the market operator must respect. Energy bids are either for consumption in charging mode $\hat{e}_{t}^{c}$ or generation in discharging mode $\hat{e}_{t}^{d}$. The ESS owner can offer upward reserve capacity by charging less $\hat{u}_{t}^{c}$ or by discharging more $\hat{u}_{t}^{d}$, and downward reserve capacity by charging more $\hat{d}_{t}^{c}$ or by discharging less $\hat{d}_{t}^{d}$ than its scheduled quantity.

2) Lower-level problem: joint energy-reserve and balancing markets: The market operator (MO) minimizes the joint

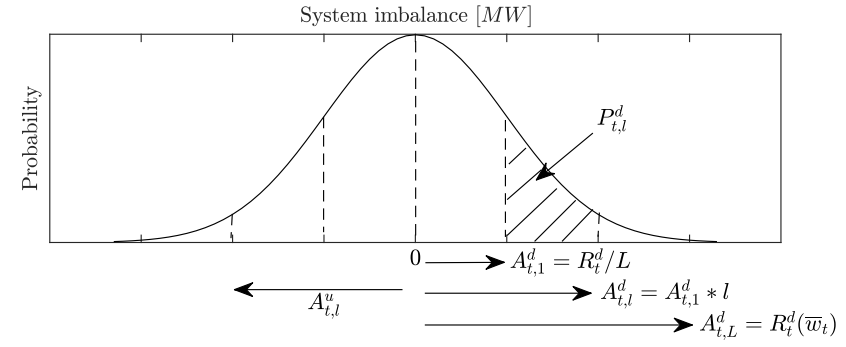

Fig. 2. The upward and downward reserve requirements are split in $L$ levels. The probability of activating scheduled reserves is calculated as the cumulative probability of reaching the level it is assigned to and all subsequent levels (i.e., levels corresponding to higher system imbalances).

energy generation, reserve procurement and expected reserve activation costs. It yields a DA dispatch schedule, the reserve procurement and possible reserve activation quantities, as well as all markets' clearing prices. The MO's problem is constrained by the ESS owner's strategic quantity bids and the ESS's technical limitations. All other market participants are assumed to be price-takers. Conventional generators offer their full capacities at average generation cost. Renewable generation is offered at $0 € / \mathrm{MWh}$ to ensure it is accepted. As a last resort, load shedding may be scheduled at the value of lost load (VOLL).

Possible system imbalances are characterized by a parametric probability density function (PDF), e.g., as a function of the total forecasted RES output. The MO uses this PDF to schedule reserve capacity, accounting for the expected reserve activation costs in the balancing market. This approach, illustrated in Fig. 2 and discussed below, builds on the probabilistic constraints proposed by Bruninx and Delarue [15].

The up- and downward reserve requirements are each split in $L$ intervals or levels with a corresponding probability of being reached $P_{t, l}^{u / d}$. This probability is computed by integrating the PDF between the interval's bounds. Procured reserves are activated in subsequent intervals in ascending order of activation costs. When a level is reached, the full reserve capacity up to its outer bound is assumed to be activated. Therefore, the probability of activation of a certain up- or downward reserve capacity is the cumulative probability of reaching its own and all subsequent levels. The resulting probabilistic reserve constraints allow determining the expected reserve activation cost when clearing the DA energy and reserve markets.

3) Assumptions: Network constraints are omitted. We assume RES forecast errors to be the only cause for real-time system imbalances. These possible imbalances are represented via a probability density function, capturing the real-time RESbased generation availability and its probability of occurrence. Operation \& maintenance or ramping costs are not considered. A cyclical boundary condition is imposed on the energy storage content of the ESS. Conventional and RES-based generation may be scheduled as upward and downward reserve provider. Conventional reserve providers may be activated at average generation cost. Emergency demand response may provide upward reserves, to be activated at the VOLL. The ESS offers generation and reserve capacity at $0 € / \mathrm{MWh}$ and demand bids at the price cap to ensure they are accepted. 


\section{B. Notation}

1) Sets:

$\mathcal{I}$

$\mathcal{J}$

$\mathcal{T}$

$\mathcal{L}$

2) Primal variables:

$\phi_{t} \quad$ Load shedding at time step $t$, MWh.

$e_{t}^{c}, e_{t}^{d} \quad$ Scheduled ESS consumption $e_{t}^{c}$ or generation $e_{t}^{d}$ in the DA market at time step $t$, MWh.

$\hat{e}_{t}^{c}, \hat{e}_{t}^{d} \quad$ ESS charge $\hat{e}_{t}^{c}$ or discharge $\hat{e}_{t}^{d}$ DA energy market bid at time step $t$, MWh.

$d_{i, t}^{g}, d_{j, t}^{w} \quad$ Procured downward reserves from generator $i$ or $j$ at time step $t$, MW.

$d_{t}^{c}, d_{t}^{d} \quad$ Procured downward ESS reserves in charge $u_{t}^{c}$ or discharging mode $u_{t}^{d}$ at time step MW.

$\hat{d}_{t}^{c}, \hat{d}_{t}^{d} \quad$ ESS downward reserve capacity bid in charge $\hat{d}_{t}^{c}$ or discharge mode $\hat{d}_{t}^{d}$ at time step $t$, MW.

$d a_{i, t, l}^{g}, d a_{j, t, l}^{w}$ Activated downward reserves from generator $i$ or $j$ at time step $t$ in level $l$, MWh.

$d a_{t, l}^{c}, d a_{t, l}^{d} \quad$ Activated downward ESS reserves in charge $d a_{t, l}^{c}$ or discharging mode $d a_{t, l}^{d}$ at time step $t$ in level $l$, MWh.

$g_{i, t} \quad$ Scheduled output of generator $i$ at time step $t$, MWh.

$u_{t}^{\phi} \quad$ Procured interruptible load contract at time step $t$, MW.

$u_{i, t}^{g}, u_{j, t}^{w} \quad$ Procured upward reserves from generator $i$ or $j$ at time step $t$, MW.

$u_{t}^{c}, u_{t}^{d} \quad$ Procured upward ESS reserves in charge $u_{t}^{c}$ or discharging mode $u_{t}^{d}$ at time step $t$, MW.

$\hat{u}_{t}^{c}, \hat{u}_{t}^{d} \quad$ ESS upward reserve capacity bid in charge $\hat{u}_{t}^{c}$ or discharge mode $\hat{u}_{t}^{d}$ at time step $t$, MW.

$u a_{t}^{\phi} \quad$ Activated interruptible load contract at time step $t$ in level $l$, MWh.

$u a_{i, t, l}^{g}, u a_{j, t, l}^{w}$ Activated upward reserves from generator $i$ or $j$ at time step $t$ in level $l$, MWh.

$u a_{t, l}^{c}, u a_{t, l}^{d}$ Activated upward ESS reserves in charge $u a_{t, l}^{c}$ or discharging mode $u a_{t, l}^{d}$ at time step $t$ in level $l$, MWh.

$v_{t} \quad$ ESS's state of charge at time step $t$, MWh.

$w_{j, t} \quad$ Scheduled output of RES-based generator $j$ at time step $t$, MWh.

3) Selected dual variables:

$\lambda_{t}^{e} \quad$ Electricity price at time step $t, € / \mathrm{MWh}$.

$\lambda_{t}^{u r}, \lambda_{t}^{d r} \quad$ Upward $\lambda_{t}^{u r}$ or downward reserve procurement price $\lambda_{t}^{d r}$ at time step $t, € / \mathrm{MW}$.

$\lambda_{t, l}^{u a}, \lambda_{t, l}^{d a} \quad$ Upward $\lambda_{t, l}^{u a}$ or downward reserve activation price $\lambda_{t, l}^{d a}$ at time step $t$ in level $l, € / M W h$.

\section{4) Parameters:}

$\eta^{c}, \eta^{d} \quad$ ESS charge $\eta^{c}$ and discharge efficiency $\eta^{d}$.

$A_{t, l}^{u}, A_{t, l}^{d} \quad$ Upward $A_{t, l}^{u}$ and downward reserve activation

volume $A_{t, l}^{d}$ at time step $t$ in level $l$, MW.

$\bar{C}, \bar{D} \quad$ ESS's charge $\bar{C}$ or discharge capacity $\bar{D}$, MW. $c_{i}^{g} \quad$ Generation cost of generator $i, € / \mathrm{MWh}$.

$c_{i}^{g, u}, c_{i}^{g, d} \quad$ Upward $c_{i}^{g, u}$ or downward reserve procurement cost $c_{i}^{g, d}$ of generator $i, € / \mathrm{MW}$.

$c^{\phi} \quad$ Value of lost load, $€ / \mathrm{MWh}$.

$c^{\phi, u} \quad$ Interruptible load contract cost, $€ / \mathrm{MW}$.

$D_{t}^{e} \quad$ Energy demand at time step $t$, MW.

$\overline{g_{i}} \quad$ Capacity of generator $i$, MW.

$P_{t, l}^{u}, P_{t, l}^{d} \quad$ Probability of reaching upward $P_{t, l}^{u}$ or downward $P_{t, l}^{d}$ reserve activation level $l$ at time step $t$.

$R_{t}^{u}, R_{t}^{d}$

Upward $R_{t}^{u}$ and downward $R_{t}^{d}$ reserve requirement at time step $t$, MW.

$\underline{v}, \bar{v} \quad$ ESS storage capacity lower $\underline{v}$ and upper bound $\bar{v}, \mathrm{MWh}$.

$\overline{w_{j, t}} \quad$ Available output of RES generator $j$ at time step $t$, MW.

\section{Mathematical Formulation}

$$
\begin{array}{lll}
\max & \sum_{t \in \mathcal{T}}\left[\lambda_{t}^{e}\left(e_{t}^{d}-e_{t}^{c}\right)+\lambda_{t}^{u r}\left(u_{t}^{d}+u_{t}^{c}\right)+\lambda_{t}^{d r}\left(d_{t}^{d}+d_{t}^{c}\right)\right. \\
& \left.+\sum_{l \in L}\left(P_{t, l}^{u} \cdot \lambda_{t, l}^{u a}\left(u a_{t, l}^{c}+u a_{t, l}^{d}\right)+P_{t, l}^{d} \cdot \lambda_{t, l}^{d a}\left(d a_{t, l}^{c}+d a_{t, l}^{d}\right)\right)\right] \\
\text { s.t. } & 0 \leq \hat{e}_{t}^{d}-\hat{d}_{t}^{d} & \forall t \in \mathcal{T}
\end{array}
$$

1) Upper-level problem (1): The ESS owner maximizes its profit from the energy $\left(\lambda_{t}^{e}\left(e_{t}^{d}-e_{t}^{c}\right)\right)$, reserve $\left(\lambda_{t}^{u r}\left(u_{t}^{d}+u_{t}^{c}\right)+\lambda_{t}^{d r}\left(d_{t}^{d}+\right.\right.$ $\left.\left.d_{t}^{c}\right)\right)$ and balancing markets $\left(\sum_{l \in L}\left(P_{t, l}^{u} \cdot \lambda_{t, l}^{u a}\left(u a_{t, l}^{c}+u a_{t, l}^{d}\right)+P_{t, l}^{d}\right.\right.$. $\left.\left.\lambda_{t, l}^{d a}\left(d a_{t, l}^{c}+d a_{t, l}^{d}\right)\right)\right)$ (Eq. (1a)). These revenues depend on the market clearing prices $\left(\lambda_{t}^{e}, \lambda_{t}^{u r}, \lambda_{t}^{d r}, \lambda_{t, l}^{u a}, \lambda_{t, l}^{d a}\right)$ and the cleared quantities $\left(e_{t}^{d}, e_{t}^{c}, u_{t}^{d}, u_{t}^{c}, d_{t}^{d}, d_{t}^{c}, u a_{t, l}^{c}, u a_{t, l}^{d}, d a_{t, l}^{c}, d a_{t, l}^{d}\right)$, which correspond to the solutions of LL problem (2). Note that, through the LL market clearing problem, the ESS owner forms a deterministic expectation of the outcome of the energy and reserve markets, whereas the balancing market is cleared for different levels of imbalances, each with their probability of occurrence $\left(P_{t, l}^{u}, P_{t, l}^{d}\right)$ (see below).

The ESS owner's decision variables are the energy $\left(\hat{e}_{t}^{d}\right.$, $\hat{e}_{t}^{c}$ ) and reserve quantities $\left(\hat{u}_{t}^{c}, \hat{u}_{t}^{d}, \hat{d}_{t}^{c}, \hat{d}_{t}^{d}\right)$ it offers, both in charging $(c)$ and $(d)$ discharging mode. These offers are constrained by (1b)-(1f), which ensure the feasibility of the non-negative energy and reserve offers considering the ESS's (dis)charging power capacity $\bar{C}$ and $\bar{D}$, and by LL problem (2). Constraint (1b) ensures that downward reserves offers in discharging mode (i.e. discharging less) never exceed the discharge offers in the energy market. Constraint (1c) limits the total discharging and upward reserve in discharging mode offers to the ESS's discharging capacity. Constraints (1d) and (1e) enforce similar limitations for charging mode. 
2) Lower-level problem (2): The MO minimizes (Eq. (2a)) the electricity generation costs in the DA electricity market (first line), the reserve procurement cost (second line) and the expected balancing costs (third line).

Constraints (2b)-(2f) are the DA electricity (2b), reserve (2c)-(2d), and balancing markets' balances (2e)-(2f). RESbased generation $\left(w_{j, t}\right)$ is offered at $0 € / \mathrm{MWh}$, whereas load shedding $\phi_{t} \geq 0$ is penalized at the VOLL $c^{\phi}$. The dual variables of $(2 \mathrm{~b})-(2 \mathrm{f})$, indicated between square brackets, may be interpreted as equilibrium prices [17]. The dual variables associated to the balancing market's power balances are probability-weighted balancing prices [18].

Cyclic constraint (2g) computes the ESS's energy content at the end of each time step $v_{t}$, assuming no activation of its procured reserves. Equations (2h) and (2i) are the worst-case reserve activation constraints proposed by Bruninx et al. [7]. These constraints ensure that the ESS owner can deliver all its procured reserves in the worst-case scenario - i.e., activation of all reserves in the upward or downward direction - without exceeding its storage content bounds $\bar{v}$ and $\underline{v}$.

The scheduled output and procured reserve quantities must respect the ESS's (dis)charging power limitations and the owner's bids (Eq. (2j)-(2k)). Similarly, the activated upward and downward reserves cannot exceed the procured reserve quantities and the owner's bids (Eq. (2l)-(2o)).

The technical limitations of the non-strategic market participants are enforced in Eq. (2p)-(2t). Constraints (2p)-(2q) limit conventional generators' power output. Similarly, constraints (2r)-(2s) are equivalent for RES-based generation. RES-based generation may provide downward reserves by additional curtailment, here limited to their positive forecast error, as RESbased generation is the only source of uncertainty. Upward RES-based reserves requires curtailing forecasted RES-based generation (Eq. (2s)).

The balancing market is cleared for $L$ distinct levels of imbalances, as discussed in Section II-A and Fig. 2. To ensure a meaningful interpretation of prevailing balancing prices, Constraints $(2 \mathrm{u})-(2 \mathrm{ac})$ enforce that all reserves which are activated in a level are also activated in all subsequent levels. Finally, Constraints (2l)-(2s) ensure all reserves that are activated in the balancing market are effectively procured in the DA reserve market.

3) Equivalent MILP problem: The bilevel optimization problem (1)-(2) is converted in an MPEC by replacing the continuous and convex LL problem (2) by the associated KKT conditions [16]. The resulting MPEC contains two types of non-linearities, i.e., (1) the UL objective function, which consists of products of LL variables, and (2) the complementary slackness conditions. The UL objective function may be reformulated using the strong duality theorem [17]. The complementary slackness conditions are recasted as a set of MILP constraints based on the big M-method [19]. The resulting MILP problem can be solved efficiently solved with off-the-shelf solvers (e.g., CPLEX). For sake of conciseness, the reformulation of the bilevel problem as an equivalent MILP problem is omitted here, but can be found in Schillemans [18].

$$
\begin{aligned}
\min & \sum_{t \in \mathcal{T}}\left[\sum_{i \in \mathcal{I}} c_{i}^{g} \cdot g_{i, t}+c^{\phi} \cdot \phi_{t}\right. \\
& +\sum_{i \in \mathcal{I}}\left(c_{i}^{g, u} \cdot u_{i, t}^{g}+c_{i}^{g, d} \cdot d_{i, t}^{g}\right)+c^{\phi, u} \cdot u_{t}^{\phi} \\
& \left.+\sum_{l \in \mathcal{L}}\left(P_{t, l}^{u}\left[\sum_{i \in \mathcal{I}} c_{i}^{g} \cdot u a_{i, t, l}^{g}+c^{\phi} \cdot u a_{t, l}^{\phi}\right]-P_{t, l}^{d} \sum_{i \in \mathcal{I}} c_{i}^{g} \cdot d a_{i, t, l}^{g}\right)\right]
\end{aligned}
$$

s.t.

$\sum_{i \in \mathcal{I}} g_{i, t}+\sum_{j \in \mathcal{J}} w_{j, t}+e_{t}^{d}=D_{t}^{e}-\phi_{t}+e_{t}^{c}, \forall t \in \mathcal{T} \quad\left[\lambda_{t}^{e}\right]$

$\sum_{i \in \mathcal{I}} u_{i, t}^{g}+u_{t}^{d}+u_{t}^{c}+\sum_{j \in \mathcal{J}} u_{j, t}^{w}+u_{t}^{\phi}=R_{t}^{u}, \forall t \in \mathcal{T}\left[\lambda_{t}^{u r}\right]$

$\sum_{i \in \mathcal{I}} d_{i, t}^{g}+d_{t}^{d}+d_{t}^{c}+\sum_{j \in \mathcal{J}} d_{j, t}^{w}=R_{t}^{d}, \forall t \in \mathcal{T}\left[\lambda_{t}^{d r}\right]$

$\sum_{i \in \mathcal{I}} u a_{i, t, l}^{g}+u a_{t, l}^{d}+u a_{t, l}^{c}+\sum_{j \in \mathcal{J}} u a_{j, t, l}^{w}+u a_{t, l}^{\phi}=A_{t, l}^{u}$,

$\forall t \in \mathcal{T}, l \in \mathcal{L}\left[P_{t, l}^{u} \cdot \lambda_{t, l}^{u a}\right]$

$\sum_{i \in \mathcal{I}} d a_{i, t, l}^{g}+d a_{t, l}^{d}+d a_{t, l}^{c}+\sum_{j \in \mathcal{J}} d a_{j, t, l}^{w}=A_{t, l}^{d}$,

$\forall t \in \mathcal{T}, l \in \mathcal{L}\left[P_{t, l}^{d} \cdot \lambda_{t, l}^{d a}\right]$

$v_{t-1}+\eta^{c} \cdot e_{t}^{c}-\frac{e_{t}^{d}}{\eta^{d}}=v_{t}, \forall t \in \mathcal{T}$

$v_{t}-\sum_{t^{*}=0}^{t}\left(u_{t^{*}}^{c} \cdot \eta^{c}+\frac{u_{t^{*}}^{d}}{\eta^{d}}\right) \geq \underline{v}, \forall t \in \mathcal{T}$

$v_{t}+\sum_{t^{*}=0}^{t}\left(d_{t^{*}}^{c} \cdot \eta^{c}+\frac{d_{t^{*}}^{d}}{\eta^{d}}\right) \leq \bar{v}, \forall t \in \mathcal{T}$

$d_{t}^{d} \leq e_{t}^{d} \leq \hat{e}_{t}^{d}, \forall t \in \mathcal{T}$

$u_{t}^{c} \leq e_{t}^{c} \leq \hat{e}_{t}^{c}, \forall t \in \mathcal{T}$

$u a_{t, L}^{d} \leq u_{t}^{d} \leq \hat{u}_{t}^{d}, \forall t \in \mathcal{T}$

$u a_{t, L}^{c} \leq u_{t}^{c} \leq \hat{u}_{t}^{c}, \forall t \in \mathcal{T}$

$d a_{t, L}^{d} \leq d_{t}^{d} \leq \hat{d}_{t}^{d}, \forall t \in \mathcal{T}$

$d a_{t, L}^{c} \leq d_{t}^{c} \leq \hat{d}_{t}^{c}, \forall t \in \mathcal{T}$

$d a_{i, t, L}^{g} \leq d_{i, t}^{g} \leq g_{i, t}, \forall i \in \mathcal{I}, t \in \mathcal{T}$

$u a_{i, t, L}^{g} \leq u_{i, t}^{g} \leq \overline{g_{i}}-g_{i, t}, \forall i \in \mathcal{I}, t \in \mathcal{T}$

$d a_{j, t, L}^{w} \leq d_{j, t}^{w} \leq w_{j, t}, \forall j \in \mathcal{J}, \forall t \in \mathcal{T}$

$u a_{j, t, L}^{w} \leq u_{j, t}^{w} \leq \overline{w_{j, t}}-w_{j, t}, \forall j \in \mathcal{J}, t \in \mathcal{T}$

$u a_{t, L}^{\phi} \leq u_{t}^{\phi} \leq R_{t}^{u}, \forall t \in \mathcal{T}$

$0 \leq u a_{i, t, l}^{g} \leq u a_{i, t, l+1}^{g}, \forall i \in \mathcal{I}, t \in \mathcal{T}, l \in \mathcal{L} \backslash\{L\}$

$0 \leq d a_{i, t, l}^{g} \leq d a_{i, t, l+1}^{g}, \quad \forall i \in \mathcal{I}, t \in \mathcal{T}, l \in \mathcal{L} \backslash\{L\}$

$0 \leq u a_{t, l}^{d} \leq u a_{t, l+1}^{d}, \forall t \in \mathcal{T}, l \in \mathcal{L} \backslash\{L\}$

$0 \leq d a_{t, l}^{d} \leq d a_{t, l+1}^{d}, \forall t \in \mathcal{T}, l \in \mathcal{L} \backslash\{L\}$

$0 \leq u a_{t, l}^{c} \leq u a_{t, l+1}^{c}, \forall t \in \mathcal{T}, l \in \mathcal{L} \backslash\{L\}$

$0 \leq d a_{t, l}^{c} \leq d a_{t, l+1}^{c}, \forall t \in \mathcal{T}, l \in \mathcal{L} \backslash\{L\}$

$0 \leq u a_{j, t, l}^{w} \leq u a_{j, t, l+1}^{w}, \forall j \in \mathcal{J}, t \in \mathcal{T}, l \in \mathcal{L} \backslash\{L\}$

$0 \leq d a_{j, t, l}^{w} \leq d a_{j, t, l+1}^{w}, \forall j \in \mathcal{J}, t \in \mathcal{T}, l \in \mathcal{L} \backslash\{L\}$

$0 \leq u a_{t, l}^{\phi} \leq u a_{t, l+1}^{\phi}, \forall t \in \mathcal{T}, l \in \mathcal{L} \backslash\{L\}$ 


\section{CASE Study}

We illustrate the model's features in a case study, based on a small power system (four conventional power plants, one RES-based generator and a single storage unit). The system imbalance follows a normal distribution with standard derivation $\bar{w}_{t} / 5$ around a zero mean. Up- and downward reserves are sized to $3 \bar{w}_{t} / 5$, providing a design reliability of $99.7 \%$. Below, we discuss several distinct features of the model and illustrate their implications on the generation dispatch, reserve procurement quantities and equilibrium prices (Fig. 3). A more rigorous discussion, including an interpretation based on the problem's equilibrium conditions, can be found in [18].

a) Inter-market trade-off: The equilibrium prices illustrate that each market participant must make inter-market trade-offs (Fig. 3). For example, at time step 8, the energy price $\lambda_{t}^{e}$ is below the generation cost of marginal unit 3 $\left(\lambda_{8}^{e}<c_{3}^{g}\right)$, which can be explained as follows. Generating an additional $M W h$ would allow generator 3 to offer this capacity as downward reserves. Because $c_{3}^{g}>c_{2}^{g}>c_{1}^{g}$, this capacity would be procured for activation in the second downward level. The resulting energy price is therefore $\lambda_{8}^{e}=c_{3}^{g}-\left(\lambda_{8}^{d r}-c_{3}^{g, d}\right)-\sum_{l=2}^{L} P_{8, l}^{d}\left(\lambda_{8, l}^{d a}+c_{3}^{g}\right)$. Note that the trade-off depends on the reserve activation probability: a higher probability of activation (i.e., lower reserve levels) increases the expected cost savings in the balancing market. For example, at time steps $2-5$, generator 1 only provides upward reserves in reserve level 2 , despite remaining head room (Fig. 3).

b) ESS's inter-temporal trade-off: The ESS's worst-case constraints imply an additional inter-temporal trade-off. Delivery of procured up- and downward reserves are ensured by virtually 'reserving' the energy to discharge or storage capacity to charge in the current and all subsequent time steps (Fig. 3(e)). These constraints implicitly define the ESS's reserve procurement cost by tightening the storage capacity's bounds for the current and all subsequent time steps. This limits the upward reserve capacity offered to the market. In this specific case, the ESS provides upward reserves for activation in level 1 throughout the day and in level 2 during the last two time steps.

c) ESS's bid strategy: The ESS owner may strategically withhold (dis)charging capacity from the energy and reserve market, which enables strategic inter-temporal and intermarket arbitrage. Moreover, the bilevel model structure allows mimicking the behavior of a profit-maximizing ESS owner exerting market power to influence all markets' prices. Note, however, that the bilevel structure implies that the ESS owner assumes an optimal reaction from the LL market clearing problem. In other words, when multiple market outcomes are possible, the ESS owner chooses the most profitable solution satisfying the LL problem's equilibrium conditions.

For example, at time step 9, generator 2 runs at full capacity and the ESS discharges. Because the ESS owner withholds all remaining capacity, generator 3 is the marginal unit, setting the energy price to $\lambda_{9}^{e}=c_{3}^{g}-\left(\lambda_{9}^{d r}-c_{3}^{g, d}\right)-\sum_{l=1}^{L} P_{9, l}^{d}\left(\lambda_{9, l}^{d a}+c_{3}^{g}\right)$.

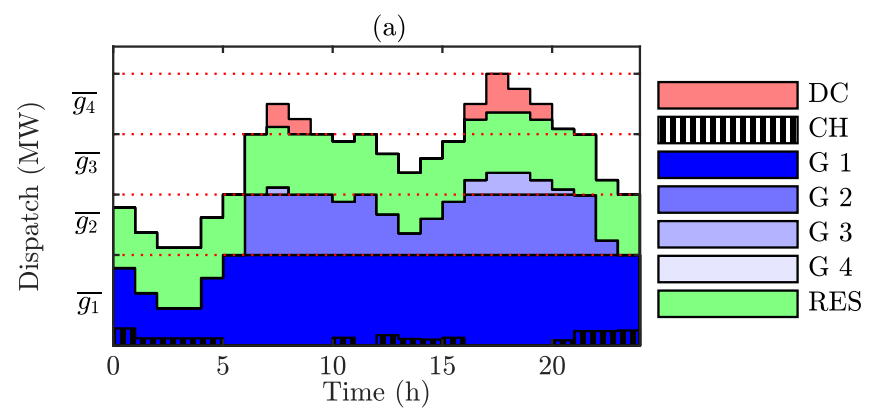

(b)

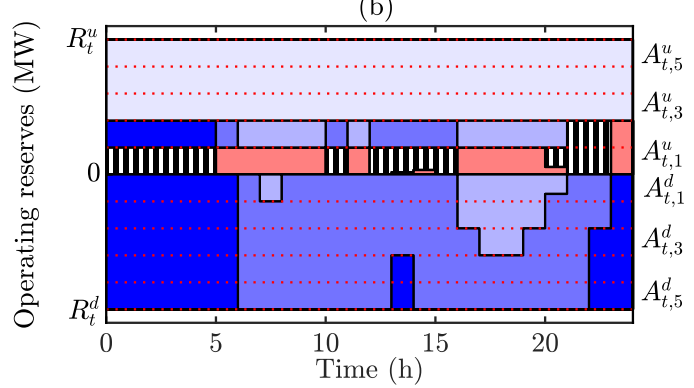

(c)
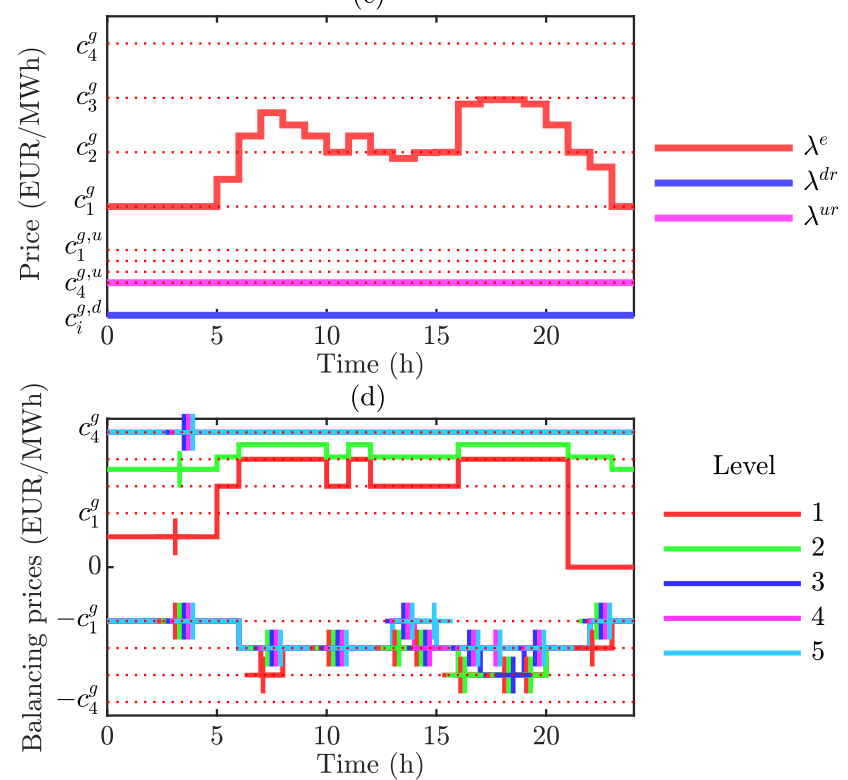

(e)

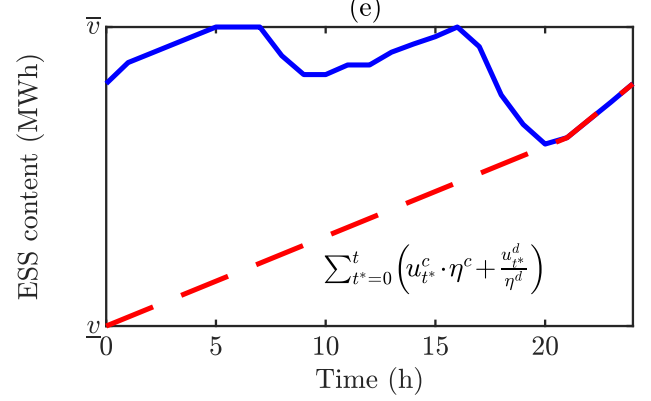

Fig. 3. (a) The scheduled generation (G), charge (CH) and discharge (DC) quantities, resulting from the day-ahead market. Dotted red lines indicate the capacity of the conventional generators. (b) The procured up- and downward reserves per market participant, ordered in order of activation in the balancing market. Dotted red lines distinguish different activation levels. (c) The energy, up- and downward reserve procurement prices. Dotted red lines represent generation and upward reserve procurement costs of the conventional generators. (d) The balancing prices per activation level. Dotted red lines represent conventional generators' generation costs. (e) Day-ahead scheduled storage content (solid line) and worst-case lower storage content bound (dashed line), reflecting the required energy content to ensure the feasibility of real-time upward ESS-based reserve activation. 
Similarly, at time step 24, generator 1 runs at full capacity and the ESS charges. The energy price $\lambda_{t}^{e}$ is not set by generator 2, i.e., the next generator in the merit order. Since the last- $M W h$ providing generator runs at full capacity, an equilibrium price range exist between that generator's and the operating costs of the next generator in the merit order, hence, the ESS owner is able to set the lowest price satisfying the LL equilibrium conditions to maximize its profit.

d) Reserve \& balancing prices: The downward reserve procurement price $\lambda_{t}^{d r}$ is always zero because $c_{i}^{g, d}=0 \forall i \in$ $\mathcal{I}$. The upward reserve procurement price $\lambda_{t}^{u r}$ is always equal to $c_{4}^{g, u r}$ since generator 4 has the lowest upward reserve procurement cost and always has headroom. The procurement of an additional $M W$ of reserves does not require its activation in the model formulation, which decouples the reserve procurement price from possible balancing market outcomes. Note that this allows that some generators do not recover their reserve procurement cost in the reserve market (e.g., generator 1 at time step 1). However, their expected profit from the balancing market counterbalances this loss: the equilibrium prices reflect an equilibrium across markets and ensure that each market participant has a non-negative expected profit.

Downward balancing prices $\lambda_{t, l}^{a d}$ are always negative because generators are willing to pay to save their generation costs. Generators with higher generation costs are activated in lower levels, thus, with a higher probability of activation, resulting in lower downward balancing prices $\lambda_{t, l}^{a d}$ for lower levels. As the ESS owner expects negative balancing prices $\lambda_{t, l}^{a d}$, it does not participate in the downward reserve market.

Upward balancing prices $\lambda_{t, l}^{a u}$ follow from the ESS owner's strategic bids, reflecting inter-temporal and inter-market tradeoffs. At time step 1, the ESS owner strategically bids $A_{1,1}^{u}$ upward reserves to set the upward balancing price in level 1 to the generation cost of the next reserve provider in the merit order (here: upward RES-based reserves). Procuring RES-based upward reserve capacity requires curtailing its scheduled generation in the energy market, which must here be compensated by additional output of generator 1 . The balancing price $\lambda_{1,1}^{u a}=\left(-c_{1}^{g, u}-\sum_{l=2}^{5} P_{1, l} c_{1}^{g}+c_{1}^{g}\right) / P_{1,1}^{u}$ hence results from the ESS owner's strategic bid and a trade-off between generation, reserve procurement and expected reserve activation costs.

\section{CONCLUSION}

Cost-effectively integrating stochastic RES-based electricity generation requires flexible assets, such as ESS. This flexibility should, however, be properly renumerated for the services it provides through the markets it participates in.

In this paper, we propose a bilevel optimization problem to study the participation of a strategic price-making ESS owner in (1) DA energy-reserve capacity and (2) RT balancing markets. This model ensures the feasibility of the realtime activation ESS-based reserves and explicitly considers the uncertainty on the balancing market outcome through probabilistic reserve constraints.
This work constitutes a first step towards a comprehensive assessment of the profitability and uptake of ESS under current and future market designs. It may be used by regulators and MOs to test market design options or could be integrated in ESS owners' daily operating procedures.

\section{ACKNOWLEDGMENT}

$\mathrm{K}$. Bruninx is a post-doctoral research fellow of the Research Foundation - Flanders (FWO) and the Flemish Institute for Technological Research (VITO).

\section{REFERENCES}

[1] J. A. Taylor, D. S. Callaway, and K. Poolla, "Competitive energy storage in the presence of renewables," IEEE Trans. Power Syst., vol. 28, no. 6, pp. $2691-2702,2013$.

[2] G. De Vivero-Serrano, K. Bruninx, and E. Delarue, "Implications of bid structures on the offering strategies of merchant energy storage systems," KU Leuven Energy Institute WP EN2018-10, 2018.

[3] X. He, E. Delarue, W. D'haeseleer, and J. M. Glachant, "A novel business model for aggregating the values of electricity storage," Energy Policy, vol. 39, no. 3, pp. 1575-1585, 2011.

[4] Y. Dvorkin, R. Fernandez-Blanco, D. S. Kirschen, H. Pandzic, J. P. Watson, and C. A. Silva-Monroy, "Ensuring Profitability of Energy Storage," IEEE Trans. Power Syst., vol. 32, no. 1, pp. 611-623, 2017.

[5] J. A. Taylor, "Financial storage rights," IEEE Trans. Power Syst., vol. 30, no. 2, pp. 997-1005, 2015.

[6] J. E. Contreras-Ocaña, M. A. Ortega-Vazquez, and B. Zhang, "Participation of an Energy Storage Aggregator in Electricity Markets," IEEE Trans. Smart Grid, vol. PP, no. 99, 2017.

[7] K. Bruninx, Y. Dvorkin, E. Delarue, D. William, and D. S. Kirschen, "Coupling Pumped Hydro Energy Storage with Unit Commitment," IEEE Trans. Sustain. Energy, vol. 7, no. 2, pp. 786-796, 2015.

[8] P. Zou, Q. Chen, Q. Xia, G. He, and C. Kang, "Evaluating the Contribution of Energy Storages to Support Large-Scale Renewable Generation in Joint Energy and Ancillary Service Markets," IEEE Trans. Sustain. Energy, vol. 7, no. 2, pp. 808-818, 2016.

[9] G. He, Q. Chen, C. Kang, P. Pinson, and Q. Xia, "Optimal Bidding Strategy of Battery Storage in Power Markets Considering PerformanceBased Regulation and Battery Cycle Life," IEEE Trans. Smart Grid, vol. 7, no. 5, pp. 2359 - 2367, 2016.

[10] R. Moreno, R. Moreira, and G. Strbac, "A MILP model for optimising multi-service portfolios of distributed energy storage," Applied Energy, vol. 137, pp. 554-566, 2015.

[11] H. Ding, P. Pinson, Z. Hu, and Y. Song, "Integrated bidding and operating strategies for wind-storage systems," IEEE Trans. Sustain. Energy, vol. 7, no. 1, pp. 163-172, 2016.

[12] H. Akhavan-Hejazi and H. Mohsenian-Rad, "Optimal operation of independent storage systems in energy and reserve markets with high wind penetration," IEEE Trans.Smart Grid, vol. 5, no. 2, pp. 1088 $1097,2014$.

[13] N. Vespermann, S. Delikaraoglou, and P. Pinson, "Offering Strategy of a Price-Maker Energy Storage System in Day-Ahead and Balancing Markets," in PowerTech IEEE, Manchester, UK, 2017.

[14] E. Nasrolahpour, J. Kazempour, H. Zareipour, and W. D. Rosehart, "A Bilevel Model for Participation of a Storage System in Energy and Reserve Markets," IEEE Trans. Sustain. Energy, vol. PP, no. 99, 2017.

[15] K. Bruninx and E. Delarue, "Endogenous Probabilistic Reserve Sizing and Allocation in Unit Commitment Models: Cost-Effective, Reliable, and Fast," IEEE Trans. Power Syst., vol. 32, no. 4, pp. 2593-2603, 2017.

[16] B. F. Hobbs and U. Helman, "Complementarity-Based Equilibrium Modeling for Electric Power Markets," in Complementarity-Based Equilibrium Modeling for Electric Power Markets, 2003, ch. 3, pp. 1-33.

[17] S. A. Gabriel, A. J. Conejo, J. D. Fuller, B. F. Hobbs, and C. Ruiz, Complementarity Modeling in Energy Markets. NYC, NY, US: Springer, 2012.

[18] A. Schillemans, "Optimal bidding strategies for large-scale energy storage system owners," Master's thesis, KU Leuven, Leuven, Belgium, 2018.

[19] J. Fortuny-Amat and B. McCarl, "A representation and economic interpretation of a two-level programming problem," JORS, vol. 32, no. 9 , pp. 783-792, 1981. 